\title{
Ingestão habitual de vitamina K em adultos e idosos
}

\author{
Habitual adult and elderly \\ intake of vitamin $\mathrm{K}$
}

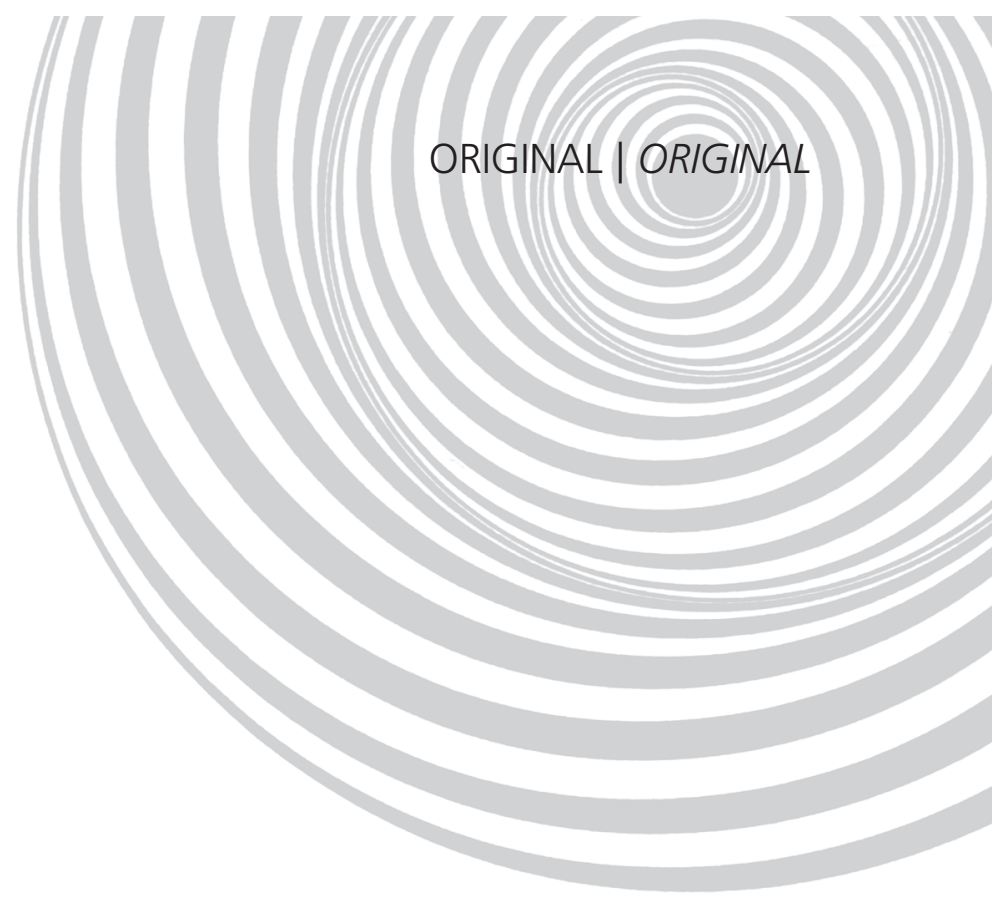

Wysllenny Nascimento de SOUZA ${ }^{1}$

Mayara Leopoldina RODRIGUES ${ }^{1}$

Marilene De Vuono Camargo PENTEADO ${ }^{1}$

RE S U M O

\section{Objetivo}

O principal objetivo deste trabalho é avaliar a ingestão habitual de vitamina $\mathrm{K}$ em adultos e idosos.

\section{Métodos}

Foi realizado um estudo transversal com a participação de 173 indivíduos de ambos os sexos. A ingestão habitual de cada participante foi avaliada pela aplicação de três recordatórios $24 \mathrm{~h}$ não consecutivos. As variabilidades intra e interpessoal foram avaliadas por meio do software PC-SIDE.

\section{Resultados}

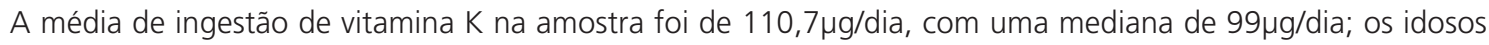
foram os que apresentaram maiores valores de ingestão, com uma mediana de $104 \mu \mathrm{g} / \mathrm{dia}$. O consumo de vitamina K entre adultos e idosos foi estatisticamente diferente $(p=0,00)$, e o consumo de hortaliças apresentou forte correlação com o consumo de vitamina K total.

\section{Conclusão}

Independentemente da faixa etária e do sexo, a ingestão de vitamina $\mathrm{K}$ foi insuficiente em quase metade da amostra, o que pode ocasionar um maior risco de inadequação dietética e acarretar problemas ósseos no futuro e/ou agravar os já presentes quando se trata da população idosa.

Termos de indexação: Adultos. Consumo alimentar. Idosos. Ingestão de alimentos. Osteoporose. Vitamina K.

A B S T R A C T

\section{Objective}

The aim of this study was to evaluate vitamin K intake by healthy adults and elderly.

\footnotetext{
${ }^{1}$ Universidade de São Paulo, Faculdade de Saúde Pública. Av. Dr. Arnaldo, 715, Cerqueira Cesar, 01246-904, São Paulo, SP, Brasil. Correspondência para/Correspondence to: WN SOUZA. E-mail: <lenny.souzaa@gmail.com>.
} 
508 WN SOUZA et al.

\section{Methods}

A cross-sectional study included 173 subjects of both genders. The habitual intake of each participant was determined by three non-consecutive 24-hour recalls. Intra- and interpersonal variability was determined by the software PC-SIDE.

\section{Results}

The mean and median vitamin $\mathrm{K}$ intakes of the sample were $110.7 \mathrm{mg} /$ day and $99 \mathrm{mg} /$ day, respectively. The elderly presented higher intakes, with a median intake of $104 \mathrm{mg} /$ day. Adult and elderly vitamin $\mathrm{K}$ intakes were statistically different $(p=0.00)$ and consumption of vegetables was strongly correlated with total vitamin $K$ intake.

\section{Conclusion}

Regardless of age and gender, vitamin K intake was inadequate in nearly half the sample, which may cause an increased risk of dietary inadequacy, possibly leading to bone disorders and/or exacerbation of those already present in the elderly.

Indexing terms: Adults. Food consumption. Aged. Eating. Osteoporosis. Vitamin K.

\section{N T R O D U Ç Ã O}

A função mais elucidada da vitamina $\mathrm{K}$ é a regulação da homeostase sanguínea, onde atua como cofator para a carboxilação de proteínas dependentes de vitamina K que catalisam o Ácido Glutâmico (Glu) para formar o Ácido $\gamma$-Carboxiglutâmico $(\mathrm{Gla})^{1,2}$. No entanto, estudos recentes têm demonstrado que essa vitamina tem múltiplas funções, principalmente no metabolismo ósseo ${ }^{3-5}$.

As mudanças decorrentes do envelhecimento populacional contribuíram para o aumento da prevalência de doenças crônicas não transmissíveis relacionadas à idade. Quando cresce a expectativa de vida da população, a osteoporose, principalmente, torna-se um problema significativo de saúde pública ${ }^{6-7}$. Dessa forma, surge um considerável interesse em relação à vitamina $K$ na prevenção da osteoporose, e a osteocalcina, uma proteína dependente de vitamina $\mathrm{K}$, tem sido um dos fundamentais alvos de estudo, pois ela está presente em altas concentrações no osso. A síntese de osteocalcina é regulada pela 1,25 - dihidroxivitamina D. No entanto, a capacidade de ligação de minerais à osteocalcina depende da gama-carboxilação dependente da vitamina $\mathrm{K}$. Como a vitamina $K$ é necessária para a carboxilação da osteocalcina, concluiu-se que concentrações insuficientes de filoquinona no plasma podem levar à mineralização óssea insuficien$\mathrm{te}^{8,9}$.

Estudos sugerem que baixas concentrações de vitamina $\mathrm{K}$ no organismo estão associadas ao seu baixo consumo, a um maior risco de fratura de quadril, menor massa óssea em idosos de ambos os sexos e a um aumento do metabolismo ósseo nas meninas $3-4,10$.

Ainda existe uma grande discrepância na ingestão de vitamina $\mathrm{K}$ em diferentes regiões e grupos etários ${ }^{11}$. Sugere-se que alguns subgrupos possam estar em risco de baixa ingestão de filoquinona, incluindo crianças $^{12}$ e idosos, principalmente aqueles com doença de Alzheimer ${ }^{13}$, e não se sabe quais são as implicações em longo prazo dessa baixa ingestão crônica de vitamina K com relação à saúde. Não há, até o momento, Ingestão Dietética Recomendada (RDA) ou Necessidade Média Estimada (EAR) estabelecidas para a vitamina K. Existe apenas a indicação da Ingestão Adequada (Al), que é o nível médio da ingestão de filoquinona usual que se presume ser adequado com base na observação de grupos de pessoas aparentemente saudáveis. A ingestão adequada de vitamina K segundo as Dietary Reference Intakes (DRI) ${ }^{14}$ está atualmente fixada em 120 $\mathrm{gg} /$ dia e $90 \mu \mathrm{g} / \mathrm{dia}$ para homens e mulheres ( $\geq 19$ anos) respectivamente.

Segundo dados apresentados pelo Institute of Medicine (IOM), os resultados que demonstram 
benefícios do uso de vitamina $\mathrm{K}$ sobre o metabolismo ósseo sugerem que o requerimento dessa vitamina para a função óssea é muito mais alto do que para manter a homeostase sanguínea ${ }^{14}$.

No Brasil, poucos trabalhos avaliaram a ingestão habitual de vitamina $\mathrm{K}$ em indivíduos saudáveis, por isso pouco se sabe sobre sua ingestão em brasileiros. Desse modo, o principal objetivo deste trabalho foi avaliar a ingestão habitual de vitamina $\mathrm{K}$ em uma população adulta e idosa.

\section{MÉ T O D O S}

Foram selecionados homens e mulheres com idade entre 21 e 81 anos; os adultos eram estudantes ou funcionários de uma universidade pública, e os idosos ( $\geq 60$ anos) eram frequentadores de um centro de referência em cidadania do idoso, ambas instituições localizadas na cidade de São Paulo, Brasil. Foram incluídos no estudo, no caso dos adultos, indivíduos saudáveis com ausência de doenças crônicas e os que não estavam fazendo algum tipo de dieta restritiva. Em relação aos idosos, foram incluídos todos aqueles que não apresentavam qualquer deficiência de cognição. Salienta-se que, no Centro de Referência, os idosos participavam de atividades físicas e socioculturais, realizadas das $9 \mathrm{~h}$ às $17 \mathrm{~h}$; dessa forma, o centro não é categorizado como uma instituição asilar, e os idosos não são institucionalizados. Foram incluídos no estudo 173 indivíduos, totalizando 519 recordatórios, em um total de 86 adultos e 87 idosos.

\section{Avaliação dietética e ingestão de vitamina $K$}

Para a coleta dos dados dietéticos e com o intuito de se conhecer a dieta habitual dos indivíduos participantes do estudo, foi utilizado o recordatório 24 horas, que foi aplicado três vezes, com um intervalo de quinze dias entre cada aplicação, sendo um desses dias um final de semana. A coleta desses dados compreendeu todas as estações do ano (novembro de 2009 a agosto de 2011).

Para diminuir os erros inerentes à aplicação do recordatório de 24 horas, foi utilizado o livro "Consumo alimentar: visualizando porções" 15 - um álbum fotográfico com alimentos e suas dimensões normais, porções e medidas-padrão, o que permitiu padronizar os dados coletados. Para o cálculo da composição de nutrientes, empregou-se a tabela online do United States Department of Agricultural (USDA) National Nutrient Database for Standard Reference, já que não existe tabela brasileira com a quantificação desse nutriente. Em algumas preparações, como sopas, molhos, farofa e panqueca, foram elaboradas fichas de preparo por meio das quais os valores totais de vitamina $\mathrm{K}$ da preparação eram obtidos mediante as quantidades da vitamina de cada ingrediente que compunha a receita. $\mathrm{O}$ mesmo procedimento foi realizado em preparações caracteristicamente brasileiras. Foram verificadas variáveis como: sexo, idade, menopausa (em muIheres), renda familiar e uso de anti-inflamatórios ou anticoagulantes, presença de osteoporose ou problemas ósseos.

\section{Análise estatística}

O método proposto pelo lowa State University (ISU) ${ }^{16}$ e incorporado no programa PC-SIDE (Software for Intake Distribution Estimation) versão 1.0, foi utilizado para obtenção das medidas descritivas de consumo de vitamina K. Nesse programa, também foram obtidos os valores de variabilidade interpessoal e intrapessoal, e a curtose da distribuição do erro de medida (quarto momento do erro intrapessoal) a partir da estimativa da distribuição da ingestão habitual de vitamina. Para determinar quais grupos de alimentos contribuíram significantemente para a ingestão total de vitamina $\mathrm{K}$, foi realizado um estudo de componentes principais.

Essa análise tem por objetivo reduzir as variáveis para que com menos componentes possa ser explicada a maior variabilidade (comporta- 
mento) dos dados. Os alimentos foram agrupados de acordo com os grupos que representam as principais fontes de vitamina K (Hortaliças $\rightarrow$ incluíam os folhosos e as leguminosas no geral; Frutas $\rightarrow$ incluíam os sucos e compotas naturais, e excluía os sucos em pó; Gorduras $\rightarrow$ composto pelos óleos, manteigas, margarinas e azeites. $\mathrm{O}$ consumo de hortaliças, frutas ou gorduras foi representado pela soma da ingestão de energia ou vitamina $\mathrm{K}$ de cada alimento pertencente ao grupo de interesse. Esse somatório foi referente aos três dias de coleta de cada indivíduo, enquanto os valores de calorias totais foram representados pela média de ingestão dos três dias para cada participante do estudo.

A análise estatística foi realizada utilizando-se o pacote estatístico $\mathrm{R}^{17}$; nos dados ajustados, a comparação entre as médias foi realizada pelo teste $t$ de Student (duas médias). Quando os critérios paramétricos (distribuição normal e homocedasticidade) não foram atingidos (dados não ajustados), os testes não-paramétricos de Mann Whitney ou Kruskal-Wallis foram utilizados. Foi adotado o nível de significância de 5\% para rejeição da hipótese de nulidade e um intervalo de confiança de $95 \%$.

O estudo foi aprovado pelo Comitê de Ética em Pesquisa da Faculdade de Ciências Farmacêuticas da Universidade de São Paulo, Protocolo $n^{\circ}$ 509/2009 em agosto de 2009. Todos os parti- cipantes assinaram um Termo de Consentimento Livre e Esclarecido antes de sua inclusão no estudo.

\section{RESULTADOS}

Os adultos representaram $49,7 \%$ do nosso estudo, e o percentual do sexo feminino foi $65,3 \%$ da população total. Apenas 19,7\% da amostra apresentavam algum tipo de doença óssea (osteopenia, osteoporose). Destes, 1,2\% era adultos e $18,5 \%$ eram idosos; entre os idosos, esse percentual era de $36,7 \%$. Problemas hemorrágicos recentes (últimos 3 meses) foram relatados por $1,2 \%$ dos entrevistados; $11 \%$ relataram o uso crônico de anti-inflamatórios ou anticoagulantes.

As características socioeconômicas da população em estudo podem ser visualizadas na Tabela 1.

A mediana de ingestão de vitamina $K$ foi de $99 \mu \mathrm{g} / \mathrm{dia}$; entre os sexos, a mediana de consumo foi de $98 \mu \mathrm{g} / \mathrm{dia}$ para o sexo masculino e $99 \mu \mathrm{g} /$ dia para o sexo feminino. Os dados sobre a variabilidade de todos os indivíduos e por sexo são apresentados na Tabela 2.

A mediana de consumo da vitamina $\mathrm{K}$ entre as faixas etárias 1,2 e 3 foi de $88 \mu \mathrm{g} / \mathrm{dia}$, $98 \mu \mathrm{g} / \mathrm{dia}$ e $104 \mu \mathrm{g} / \mathrm{dia}$, respectivamente. O consumo de vitamina $\mathrm{K}$ foi estatisticamente diferente

Tabela 1. Características socioeconômicas de adultos da Faculdade de Ciências Farmacêuticas da Universidade de São Paulo e idosos do Centro de Referência em Cidadania do Idoso da Cidade de São Paulo nos anos de 2010-2011.

\begin{tabular}{|c|c|c|c|c|c|c|c|}
\hline \multicolumn{2}{|c|}{ Características } & \multicolumn{6}{|c|}{ Faixas etárias } \\
\hline & & \multicolumn{2}{|c|}{1 (20- 29 anos) } & \multicolumn{2}{|c|}{2 (30- 59 anos) } & \multicolumn{2}{|c|}{3 ( $\geq 60$ anos) } \\
\hline & & $n$ & $\%$ & $n$ & $\%$ & $n$ & $\%$ \\
\hline \multirow{4}{*}{ Sexo } & Masculino & 17 & 9,8 & 15 & 8,7 & 28 & 16,2 \\
\hline & Feminino & 32 & 18,5 & 22 & 12,7 & 59 & 34,1 \\
\hline & Média* & \multicolumn{2}{|c|}{$23,9, \mathrm{DP}=2,3$} & \multicolumn{2}{|c|}{$42,2, \mathrm{DP}=10,1$} & \multicolumn{2}{|c|}{$68,7, \mathrm{DP}=6,18$} \\
\hline & 1-3 salários & 22 & 12,7 & 9 & 5,2 & 51 & 29,6 \\
\hline \multirow{3}{*}{ Renda\$ } & $>3$ até 6 salários & 13 & 7,5 & 17 & 9,8 & 27 & 15,6 \\
\hline & $>6$ até 8 salários & 2 & 1,2 & 6 & 3,5 & 3 & 1,7 \\
\hline & > 8 salários & 12 & 6,9 & 5 & 2,9 & 6 & 3,5 \\
\hline
\end{tabular}

*Média geral da idade por faixa etária; ' $R$ Renda familiar expressa em salários-mínimos (1/4/2010 a 31/1/2011) = R\$510,00. DP: Desvio-Padrão. 
Tabela 2. Média, mediana, desvio-padrão e componentes de variância na ingestão de vitamina K em adultos da Faculdade de Ciências Farmacêuticas da Universidade de São Paulo e idosos do Centro de Referência em Cidadania do Idoso de São Paulo no ano de 2010-2011.

\begin{tabular}{lcccc}
\hline & Homens $^{\mathbf{a}}$ & Mulheres $^{\mathbf{a}}$ & Idosos $^{\mathbf{b}}$ & Total $^{\mathbf{c}}$ \\
\hline Média $(\mu \mathrm{g} / \mathrm{dia})$ & 109,6 & 111,9 & 126,5 & 110,7 \\
Mediana $(\mu \mathrm{g} / \mathrm{dia})$ & 98 & 99 & 104 & 99 \\
P25 $(\mu \mathrm{g} / \mathrm{dia})$ & 72 & 71 & 70 & 72 \\
P90 $(\mu \mathrm{g} /$ dia $)$ & 179 & 186 & 228 & 180 \\
Desvio-padrão $(\mu \mathrm{g} /$ dia) & 54 & 58,5 & 85,3 & 55,8 \\
Variabilidade Intrapessoal & 0,802 & 0,786 & 0,723 & 0,790 \\
Variabilidade Interpessoal & 0,230 & 0,220 & 0,288 & 0,220 \\
\hline
\end{tabular}

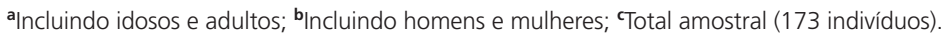

entre as faixas etárias 1 e $3(p=0,0001)$ e entre as faixas etárias 2 e $3(p=0,008)$ (Figura 1).

Quanto ao consumo de energia em relação à ingestão de hortaliças e de frutas, observou-se que ele foi maior nos idosos (Figura 2). O consumo de energia através da ingestão de hortaliças foi significantemente diferente entre os adultos jovens e os idosos $(p=0,04)$ e maior nas mulheres do que nos homens $(p=0,00)$. Em contraposição, o consumo de energia através da ingestão de frutas foi discretamente maior nos idosos, entretanto não foi estatisticamente significante entre as faixas etárias.

Em relação aos grupos de alimentos mais importantes na ingestão total da vitamina $\mathrm{K}$, foi

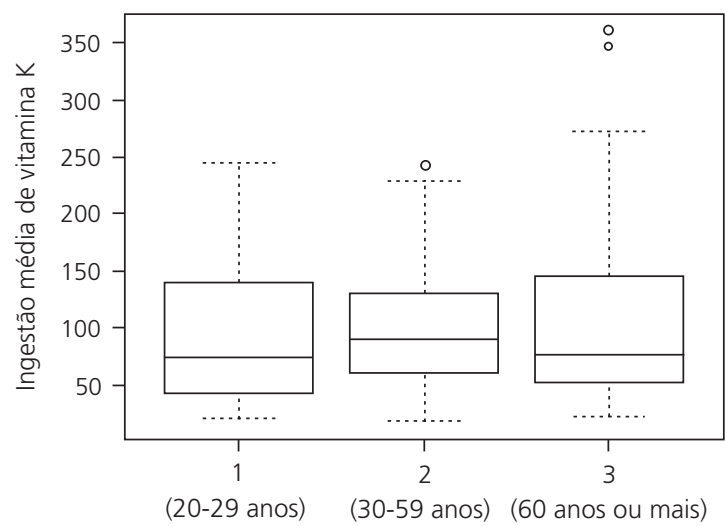

Figura 1. Consumo médio de vitamina K em adultos da Faculdade de Ciências Farmacêuticas da Universidade de São Paulo e idosos do Centro de Referência em Cidadania do Idoso de São Paulo no ano de 2010-2011. constatado que a ingestão total dessa vitamina através do consumo de gorduras foi diferente estatisticamente entre as faixas etárias $(p=0,04)$, e essa diferença foi mais acentuada entre as faixas etárias 1 e $3(p=0,006)$. Não houve diferença estatística entres os sexos em relação à ingestão total de vitamina $K$ nem entre as faixas etárias em relação ao consumo de hortaliças. O mesmo ocorreu quando considerado o consumo de frutas entre os sexos e entre as faixas etárias $(p=0,64)$ e $(p=0,28)$, respectivamente.

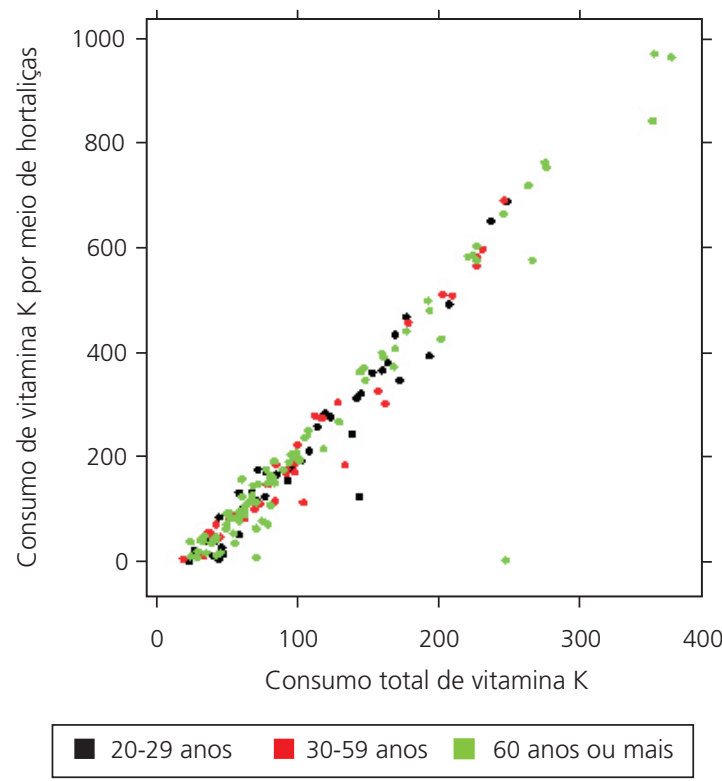

Figura 2. Diagrama de dispersão do consumo de vitamina K através da ingestão de hortaliças, segundo a faixa etária. São Paulo (SP), 2011. 
Houve uma correlação linear entre o consumo total de vitamina $\mathrm{K}$ e a ingestão dessa vitamina através de hortaliças; observou-se ainda que o consumo de vitamina $K$ através da ingestão de hortaliças apresentou forte correlação com o consumo total de vitamina $K(0,95)$ e baixa correlação com o consumo de frutas $(0,28)$ e gorduras $(0,22)$ (Figura 3).

Não houve significância estatística na ingestão de vitamina $K$ em relação às estações do

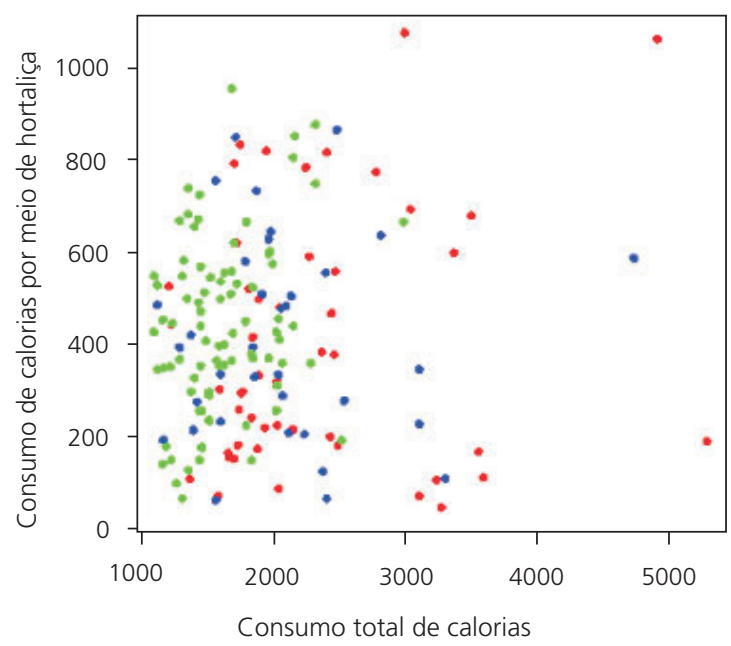

20-29 anos 30-59 anos 60 anos ou mais

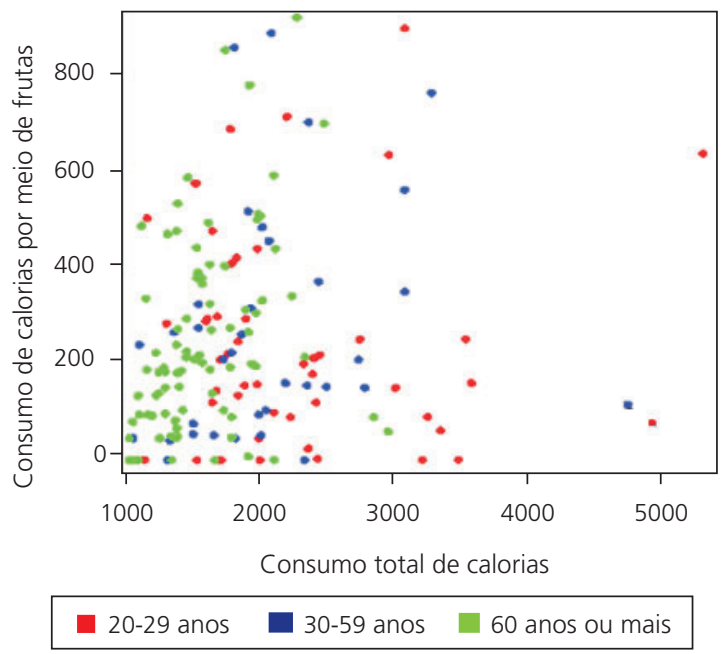

Figura 3. Diagrama de dispersão do consumo de energia através da ingestão de hortaliças e frutas segundo a faixa etária em adultos e idosos. São Paulo (SP), 2011. ano. O consumo foi semelhante entre as estações, apresentando uma mediana de ingestão de $95,12 \mu \mathrm{g} /$ dia no verão, $74,33 \mu \mathrm{g} / \mathrm{dia}$ no outono, $78,29 \mu \mathrm{g} /$ dia no inverno e $71,22 \mu \mathrm{g} /$ dia na primavera.

\section{I S C U S S Ã O}

Atualmente vem crescendo o número de estudos epidemiológicos que enfocam as possíveis relações entre ingestão de vitamina $\mathrm{K}$ e Doenças Crônicas Não-Transmissíveis (DNCT), principalmente doenças cardiovasculares ${ }^{18}$ e saúde óssea ${ }^{10}$. Tais estudos evidenciam a importância da ingestão adequada dessa vitamina. Na população estudada, independentemente do sexo e da faixa etária, o percentual de ingestão de vitamina $\mathrm{K}$ acima da Al foi baixo. O Institute of Medicine preconiza que para uma menor prevalência de inadequação, o consumo da referida vitamina deve ser $\geq$ Al. Similarmente aos nossos resultados, Pinheiro et al. ${ }^{6}$ também encontraram um baixo percentual de ingestão de vitamina $\mathrm{K}$ acima da recomendação de acordo com o sexo e a idade.

De modo geral, em nosso estudo, as mulheres apresentaram um maior consumo de vitamina $\mathrm{K}$ em relação aos homens: resultados semelhantes foram encontrados em outros estudos, em americanos ${ }^{4}$ e em brasileiros ${ }^{6}$. Nesse último estudo, a mediana de ingestão de vitamina $K$ foi menor do que os valores que encontramos em nossa amostra; em contraposição, outros autores relatam valores de ingestão superiores aos que encontramos ${ }^{19-21}$. Essas diferenças podem depender das discrepâncias quanto às tabelas de composição de alimentos para vitamina K. É plausível também que ocorram diferenças regionais, culturais e econômicas na ingestão de alimentos fontes de vitamina $\mathrm{K}$ que podem explicar as diferenças dos resultados.

Em relação à ingestão por faixa etária, a mediana de consumo de vitamina $\mathrm{K}$ foi maior entre os idosos, e essa diferença foi significativa principalmente entre os adultos jovens e os idosos. Os trabalhos reunidos por Booth \& Suttie et al. ${ }^{22}$ indicam que a ingestão média por parte de adultos 
jovens é de 60-110 $\mathrm{g} / \mathrm{dia}$, e por parte dos idosos é de 80-210 $\mathrm{g} / \mathrm{dia}$. Recente estudo com a população idosa na Holanda também revelou uma alta ingestão de vitamina $\mathrm{K}$ (filoquinona) com média de $200 \mu \mathrm{g} / \mathrm{dia}^{18}$; em outros estudos com a população idosa, os resultados foram semelhantes aos deste trabalho, em que se verifica também um consumo de vitamina $K$ mais elevado ${ }^{20,23}$. Esse resultado pode ser atribuído a um maior consumo de hortaliças e a um menor consumo de alimentos processados por essa população ${ }^{24}$. Com uma população semelhante à deste estudo - adultos e idosos -, Booth et al. ${ }^{25}$ observaram que não houve diferença significativa quanto à ingestão de vitamina $\mathrm{K}$ por idade, embora os valores medianos de ingestão de vitamina $\mathrm{K}$ fossem maiores nos idosos.

Em relação aos componentes de variância no consumo de vitamina $\mathrm{K}$, em um recente estudo com a população idosa, foi verificada uma variância interpessoal de 0,186 e uma variância intrapessoal de $0,587^{26}$. Os dados encontrados no referido estudo diferem dos encontrados por esta pesquisa: a maior diferença foi nos valores de variância intrapessoal, que podem ser resultante das diferenças nutricionais e culturais da população em questão e implicam também que a precisão da estimativa do consumo habitual de um indivíduo, obtida por apenas três medidas, como no caso do presente estudo, é mais baixa do que a obtida por Presse et al. ${ }^{26}$, que utilizou a aplicação de seis recordatórios 24h. A alta variância intrapessoal encontrada em nossos resultados provavelmente resulte da grande quantidade de vitamina $\mathrm{K}$ encontrada em alguns alimentos consumidos episodicamente, como folhosos verdes. A baixa variância interpessoal encontrada no presente estudo pode em parte ser explicada pela relativa homogeneidade da amostra (idosos saudáveis e com relativo poder de compra). Presse et al. ${ }^{26}$ também encontraram valores baixos para a variância interpessoal em seu estudo.

Com relação à variabilidade entre os sexos, observou-se que os homens apresentavam maior variabilidade intrapessoal do que as mulheres; alguns autores relataram o inverso em relação a outros nutrientes ${ }^{27,28}$. Entretanto, isso não altera os resultados deste estudo, visto que foi muito pequena a diferença encontrada entre homens e mulheres. Ressalta-se que a redução dos efeitos da variabilidade intrapessoal, e consequentemente melhor precisão da estimativa de ingestão habitual, pode ser alcançada somente aumentando o número de coletas para um mesmo indivíduo, ou seja, não pelo aumento do tamanho da amostra ${ }^{29}$ e sim pelo aumento do número de replicações das medidas dietéticas ${ }^{30}$, como realizado no presente estudo.

Os resultados encontrados na população brasileira mostram que o consumo de vegetais no geral é bastante baixo ${ }^{31-33}$. Entretanto, vários estudos relatam que o consumo de frutas, hortaliças e legumes é maior na população idosa ${ }^{34,35}$, assim como o encontrado no presente estudo, em que foi constatado que o consumo de hortaliças e frutas é maior na população idosa.

O grupo de hortaliças foi o que mais contribuiu para a ingestão total de vitamina K: essa contribuição foi igual tanto por sexo quanto por idade. Resultados semelhantes foram descritos por Booth et al. ${ }^{24}$ que observaram que, na ingestão habitual, foram as hortaliças que mais contribuíram para ingestão total de vitamina $\mathrm{K}^{24}$. A ingestão de vitamina $K$ total através do consumo de gorduras foi menor do que a referida por essa autora: isso pode ter ocorrido pelo fato de que no presente estudo não foram incluídos no grupo de gorduras alimentos como molhos gordurosos, snacks, tortas, bolos e outros alimentos ricos em gordura e que contribuem para a ingestão total da referida vitamina.

Não foi observada significância estatística na ingestão de vitamina $\mathrm{K}$ em relação às estações do ano, tal como descrito por outros estudos ${ }^{24,36,37}$. Contudo, Manotti et al.38, estudando o efeito da sazonalidade na terapia anticoagulante, relatou que ele foi reduzido durante o verão e a primavera em relação ao outono e ao inverno ${ }^{38}$. Eles atribuíram esse resultado à maior disponibilidade de vitamina $\mathrm{K}$ na dieta através do consumo elevado de vegetais frescos nas estações mais quentes. Presse et al. ${ }^{26}$ também encontraram variação no consumo de vitamina $\mathrm{K}$ em relação à sazonali- 
dade; eles também atribuem os resultados à maior disponibilidade e aos preços mais baixos de legumes frescos durante os meses de primavera e verão, especialmente em vegetais de folhas verdes. Apesar disso, eles concluem que o dia da semana e a estação do ano explicaram uma insignificante porção da variância intrapessoal no seu estudo e afirmam que a ingestão de vitamina $\mathrm{K}$ pode, portanto, ser avaliada independentemente do dia ou da estação do $a n o^{26}$.

\section{CONCLUS Ã O}

A ingestão habitual de vitamina $\mathrm{K}$ foi maior nos idosos de ambos os sexos, e isso esteve altamente relacionado com uma maior ingestão de vegetais. Entretanto, neste estudo, a população idosa ainda apresenta uma baixa ingestão de vitamina $\mathrm{K}$ e consequentemente maior risco de inadequação dietética.

\section{COLABORADORES}

WN SOUZA participou da coleta de dados e da redação do artigo. ML RODRIGUES participou da coleta de dados. MVC PENTEADO participou da supervisão da coleta de dados e da redação do artigo.

\section{REFERÊ NCIAS}

1. Booth SL. Roles for vitamin K beyond coagulation. Annu Rev Nutr. 2009; 29(1):89-110. doi: 10.1146/ annurev-nutr-080508-141217.

2. Davidson KW, Sadowski JA. Determination of vitamin $\mathrm{K}$ compounds in plasma or serum by highperformance liquid chromatography using postcolumn chemical reduction and fluorimetric detection. Methods Enzymol. 1997; 282:408-21. doi: 10.1016/S0076-6879(97)82124-6.

3. Cockayne S, Adamson J, Lanham-New S, Shearer MJ, Gilbody S, Torgerson DJ. Vitamin K and the prevention of fractures: systematic review and meta-analysis of randomized controlled trials. Arch Intern Med. 2006; 166(12):1256-61. doi: 10.1001/ archinte.166.12.1256.

4. Booth SL, Broe KE, Peterson JW, Cheng DM, Dawson-Hughes B, Gundberg CM, et al. Associations between vitamin $\mathrm{K}$ biochemical measures and bone mineral density in men and women. J Clin Endocrinol Metab. 2004; 89(10): 4904-9. doi: 10.1210/jc.2003-031673.

5. Truong JT, Booth SL. Emerging issues in vitamin $\mathrm{K}$ research. J Evidence-Based Compl Alter Med. 2011; 16(1):73-9. doi: 10.1177/1533210110392953.

6. Pinheiro MM, Schuch NJ, Genaro PS, Ciconelli RM, Ferraz MB, Martini LA. Nutrient intakes related to osteoporotic fractures in men and women-the Brazilian Osteoporosis Study (BRAZOS). Nutr J. 2009; 8(6):1-8 doi: 10.1186/1475-2891-8-6.

7. Ferrari, SL. Osteoporosis: a complex disorder of aging with multiple genetic and environmental determinants. In: Simopoulos AP, organizador. World review of nutrition and dietetics. Basel: Karger; 2005. p.35-51.

8. Yaegashi Y, Onoda T, Tanno K, Kuribayashi T, Sakata $\mathrm{K}$, Orimo $\mathrm{H}$. Association of hip fracture incidence and intake of calcium, magnesium, vitamin $D$, and vitamin K. Eur J Epidemiol. 2008; 23(3):219-25. doi: 10.1007/s10654-008-9225-7.

9. Bolton-Smith C, McMurdo MET, Paterson CR, Mole PA, Harvey JM, Fenton ST, et al. Two-year randomized controlled trial of vitamin $\mathrm{K}_{1}$ (phylloquinone) and vitamin $D_{3}$ plus calcium on the bone health of older women. J Bone Miner Res. 2007; 22(4):509-19. doi: 10.1359/JBMR.0701 16.

10. Macdonald HM, McGuigan FE, Lanham-New SA, Fraser WD, Ralston SH, Reid DM. Vitamin $\mathrm{K}_{1}$ intake is associated with higher bone mineral density and reduced bone resorption in early postmenopausal Scottish women: no evidence of gene-nutrient interaction with apolipoprotein E polymorphisms. Am J Clin Nutr. 2008; 87(5):1513-20.

11. Booth SL, Al Rajabi A. Determinants of vitamin K status in humans. Vitam Horm. 2008; 78:1-22. doi: 10.1016/S0083-6729(07)00001-5.

12. Prynne CJ, Thane CW, Prentice A, Wadsworth MEJ. Intake and sources of phylloquinone (vitamin $\mathrm{K}(1)$ ) in 4-year-old British children: comparison between 1950 and the 1990s. Public Health Nutr. 2005; 8(2):171-80. doi: 10.1079/PHN2004674.

13. Presse N, Shatenstein B, Kergoat M-J, Ferland G. Low vitamin $\mathrm{K}$ intakes in community-dwelling elders at an early stage of Alzheimer's disease. J Am Diet Assoc. 2008; 108(12):2095-9. doi: 10.1016/j.jada. 2008.09.013.

14. Institute of Medicine. Food and Nutrition Board. Dietary reference intakes for vitamin $A$, vitamin $\mathrm{K}$, arsenic boron, chromium, copper, iodine, iron, manganese, molybdenum, nickel, silicon, vanadium, and zinc. Washington: National Academy Press; 2001.

15. Monteiro JP. Consumo alimentar visualizando porções. Rio de Janeiro: Guanabara Koogan; 2007. 
16. Nusser SM, Carriquiry AL, Dodd KW, Fuller WA. A semiparametric transformation approach to estimating usual daily intake distributions. J Am Stat Assoc. 1996; 91(436):1440-9. doi: 10.2307/ 2291570.

17. Development Core Team. R: a language and environment for statistical computing [Internet]. Viena; 2005 [cited 2011 Oct 20]. Available from: <http: www.R-project.org>.

18. Beulens JWJ, van der A Daphne L, Grobbee DE, Sluijs I, Spijkerman AMW, van der Schouw YT. Dietary phylloquinone and menaquinones intakes and risk of type 2 diabetes. Diabetes Care. 2010; 33(8):1699-705. doi: 10.2337/dc09-2302.

19. Nimptsch K, Nieters A, Hailer S, Wolfram G, Linseisen J. The association between dietary vitamin $\mathrm{K}$ intake and serum undercarboxylated osteocalcin is modulated by vitamin $\mathrm{K}$ epoxide reductase genotype. Br J Nutr. 2009; 101(12):1812-20. doi: $10.1017 /$ S0007114508131750.

20. McKeown NM, Jacques PF, Gundberg CM, Peterson JW, Tucker KL, Kiel DP, et al. Dietary and nondietary determinants of vitamin $\mathrm{K}$ biochemical measures in men and women. J Nutr. 2002; 132(6):13290-34.

21. Feskanich D, Weber P, Willett WC, Rockett $H$, Booth $\mathrm{SL}$, Colditz GA. Vitamin K intake and hip fractures in women: a prospective study. Am J Clin Nutr. 1999; 69(1):74-9.

22. Booth SL, Suttie JW. Dietary intake and adequacy of vitamin K. J Nutr. 1998; 128(5):785-8.

23. Bulló $M$, Estruch $R$, Salas-Salvadó J. Dietary vitamin $\mathrm{K}$ intake is associated with bone quantitative ultrasound measurements but not with bone peripheral biochemical markers in elderly men and women. Bone. 2011; 48(6):1313-8. doi: 10.1016/ j.bone.2011.03.767.

24. Booth SL, Pennington JA, Sadowski JA. Food sources and dietary intakes of vitamin K-1 (phylloquinone) in the American diet: data from the FDA Total Diet Study. J Am Diet Assoc. 1996; 96(2):149-54. doi: 10.1016/S0002-8223(96)000 44-2.

25. Booth SL, Tucker KL, McKeown NM, Davidson KW, Dallal GE, Sadowski JA. Relationships between dietary intakes and fasting plasma concentrations of fat-soluble vitamins in humans. J Nutr. 1997; 127(4):587-92.

26. Presse N, Payette $H$, Shatenstein B, Greenwood CE, Kergoat $M-J$, Ferland $G$. A minimum of six days of diet recording is needed to assess usual vitamin $\mathrm{K}$ intake among older adults. J Nutr. 2011; 141(2): 341-6. doi: 10.3945/jn.110.132530.

27. Nelson M, Black AE, Morris JA, Cole TJ. Betweenand within-subject variation in nutrient intake from infancy to old age: estimating the number of days required to rank dietary intakes with desired precision. Am J Clin Nutr. 1989; 50(1):155-67.
28. Palaniappan U, Cue RI, Payette H, Gray-Donald K. Implications of day-to-day variability on measurements of usual food and nutrient intakes. J Nutr. 2003; 133(1):232-5.

29. Sempos CT, Looker AC, Johnson CL, Woteki CE. The importance of withing -person variability in estimating prevalence. In: MacDonald I. Monitoring dietary intakes. New York: Springer-Verlag; 1991. p.99-109.

30. Willett WC. Future directions in the development of food-frequency questionnaires. Am J Clin Nutr. 1994; 59(1 Suppl):171S-4S.

31. Instituto Brasileiro de Geografia e Estatística. Pesquisa de orçamentos familiares 2008-2009: análise do consumo alimentar pessoal no Brasil [Internet]. Rio de Janeiro: IBGE; 2010 [acesso 2011 ago 8]. Disponível em: <http://www.ibge.gov.br/ home/estatistica/populacao/condicaodevida/pof/ 2008_2009_analise_consumo/pofanalise_2008_ 2009.pdf>.

32. Jaime PC, Machado FMS, Westphal MF, Monteiro CA. Educação nutricional e consumo de frutas e hortaliças: ensaio comunitário controlado. Rev Saúde Pública. 2007; 41(1):154-7. doi: 10.1590/S 0034-89102006005000014.

33. Viebig RF, Pastor-Valero M, Scazufca M, Menezes PR. Consumo de frutas e hortaliças por idosos de baixa renda na cidade de São Paulo. Rev Saúde Pública. 2009; 43(5):806-13. doi: 10.1590/S003489102009005000048.

34. Figueiredo ICR, Jaime PC, Monteiro CA. Fatores associados ao consumo de frutas, legumes e verduras em adultos da cidade de São Paulo. Rev Saúde Pública. 2008; 42(5):777-85. doi: 10.1590/S 0034-89102008005000049.

35. Jaime PC, Monteiro CA. Consumo de frutas e hortaliças na população adulta brasileira, 2003. Cad Saúde Pública. 2005; 21(Suppl 1):S19-S24. doi: 10.15 90/S0102-311X2005000700003.

36. Thane CW, Paul AA, Bates CJ, Bolton-Smith C, Prentice $A$, Shearer MJ. Intake and sources of phylloquinone (vitamin K1): variation with sociodemographic and lifestyle factors in a national sample of British elderly people. Br J Nutr. 2002; 87(6):605-13. doi: 10.1079/BJN2002583.

37. Price R, Fenton S, Shearer MJ, Bolton-Smith C. Daily and seasonal variation in phylloquinone (vitamin K1) intake in Scotland [abstract]. Proc Nutr Soc (New Zeland). 1996; 55 244A.

38. Manotti C, Quintavalla R, Pattacini C, Pini M. Seasonal variation of oral anticoagulant effect. Thromb Haemost. 1994; 71(6):802-3.

Recebido em: 24/1/2012 Versão final em: 15/5/2012 Aprovado em: 6/6/2012 
